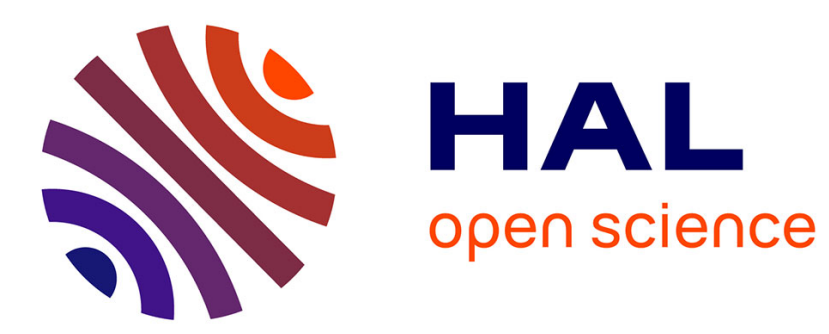

\title{
Discrete element method to simulate continuous material by using the cohesive beam model
}

Damien Andre, Ivan Iordanoff, Jean-Luc Charles, Jérôme Neauport

\section{To cite this version:}

Damien Andre, Ivan Iordanoff, Jean-Luc Charles, Jérôme Neauport. Discrete element method to simulate continuous material by using the cohesive beam model. Computer Methods in Applied Mechanics and Engineering, 2012, 213, p.113-125. 10.1016/j.cma.2011.12.002 . hal-00748669

\section{HAL Id: hal-00748669 \\ https://hal.science/hal-00748669}

Submitted on 5 Nov 2012

HAL is a multi-disciplinary open access archive for the deposit and dissemination of scientific research documents, whether they are published or not. The documents may come from teaching and research institutions in France or abroad, or from public or private research centers.
L'archive ouverte pluridisciplinaire HAL, est destinée au dépôt et à la diffusion de documents scientifiques de niveau recherche, publiés ou non, émanant des établissements d'enseignement et de recherche français ou étrangers, des laboratoires publics ou privés. 


\title{
Discrete element method to simulate continuous material by using the cohesive beam model
}

\author{
Damien André $^{\mathrm{a}}$, Ivan Iordanoffa ${ }^{\mathrm{a}}$, Jean-luc Charles ${ }^{\mathrm{a}}$, Jérôme Néauport ${ }^{\mathrm{b}}$ \\ ${ }^{a}$ Arts $\mathcal{G}$ Métiers ParisTech, I2M-DuMAS, UMR 5295 CNRS F-33405, Talence, France \\ ${ }^{b}$ Commissariat à l'Énergie Atomique, Centre d'Études Scientifiques et Techniques d'Aquitaine, BP 2, 33114 Le Barp, France
}

\begin{abstract}
The mechanical behavior of materials is usually simulated by the continuous mechanics approach. However, simulation of non-continuous phenomena like multi-fracturing is not well adapted to a continuous description. In this case, the discrete element method (DEM) is a good alternative because it naturally takes into account discontinuities.

Many researchers have shown interest in this approach for wear and fracture simulation. The problem is that, while DEM is well adapted to simulate discontinuities, it is not suitable to simulate continuous behavior. In problems of wear or fracture, material is composed of continuous parts and discontinuous interfaces. The aim of the present work is to improve the ability of DEM to simulate the continuous part of the material using cohesive bond model.

Continuous mechanics laws cannot be used directly within a DEM formulation. A second difficulty is that the volume between the discrete elements creates an artificial void inside the material. This paper proposes a methodology that tackles these theoretical difficulties and simulates, using a discrete element model, any material defined by a Young's modulus, Poisson's ratio and density, to fit the static and dynamic mechanical behavior of the material. The chosen cohesive beam model is shown to be robust concerning the influence of the discrete element sizes. This method is applied to a material which can be considered as perfectly elastic: fused silica.
\end{abstract}

Keywords: Discrete element method, DEM, Calibration, Elastic, Dynamic, Fused silica

\section{Introduction}

The discrete element method (DEM) can describe quite naturally a granular medium. However, the number of discrete elements to manage is high and it requires computational resources. The development of this method began in the early 1980s (1). More recently, researchers have used this method to study the damage of heterogeneous solids such as concrete (2) or rock (3), and homogeneous materials such as ceramics (4).

Discrete element model is well adapted to simulate a media that has a great number of interfaces. It has been widely used to study tribological problems like wear phenomena. In this kind of problem, the material has a continuous part (the volume above the surface that is not yet affected by the wear), a continuous part with cracks (called subsurface damage in abrasion process terminology) and a discontinuous part (the interfacial media, called third body, that is a mixture of abrasive particles and wear particles). Discrete element model must be able to simulate with accuracy all these parts of the material. Unlike continuous approaches, the main difficulty for DEM is to simulate properly the continuous material.

This paper focuses on a material which can be consideblue as homogeneous, isotropic and perfectly elastic: fused silica. This work is a continuation of a previous study that investigated subsurface damage in silica glass due to surface polishing (5). In this previous study, discrete element models have shown qualitatively good agreement with experiments. The challenge, now, is to propose a 3D DEM spherical model allowing quantitative results for the silica considered as a continuous media.

A preliminary task is the cohesive bond choice to model correctly the subsurface damage layer during the silica abrasive process. There are two main approaches to DEM : the dual spring model (a pair of normal and tangential springs) $(6,7,8,9)$ and the cohesive beam model $(10,11,12)$. The beam cohesive model is not as well established in the literature as the classical dual spring model. Therefore, Schlangen and Garboczi in $(10, \S 3)$ have shown that the beam cohesive bond produces more realistic crack pattern than the simple spring 
model or the dual spring model. A further work of these authors $(11, \S 3)$ have studied the influence of the initial geometric arrangement. They conclude that a disordered configuration gives more realistic crack geometry than an ordered geometrical configuration.

According to these results, the discrete element approach used in this paper is quite similar to the model described by Carmona in (12). An initial spherical domain is generated by a numerical compaction method. A dispersion applied to the discrete element radii allows random geometrical arrangement. Then, the discrete elements are connected by the "beam cohesive bonds".

The proposed model must simulate static and dynamic behavior characterized by Young's modulus, Poisson's ratio, mechanical wave celerity and natural frequencies.

The difficulty is that the mechanical behavior of a structure composed of a large number of discrete elements cannot be analytically predicted. Global behaviors are the result of a large number of interactions between discrete elements and can be considered as an emergent physical property (13, preface). Implicitly, two scales are considered in a discrete element approach:

- the structure scale, represented by a set of discrete elements. This scale will be called the "macroscopic scale".

- the discrete element scale and its elementary interaction with its neighbors. This scale will be called the "microscopic scale".

Note that the interesting properties, e.g., the Young's modulus, the Poisson's ratio, mechanical wave celerity and natural frequencies should be considered, in the DEM model, as emergent properties at the macroscopic scale. Furthermore, unlike the finite element method (14), continuous mechanical behavior laws cannot be directly introduced into the DEM formulation. As a result, the difficulty is to quantify DEM microscopic interaction laws according to continuous mechanical behavior.

This problema has been discussed in detail by OstojaStarzewski (15). The author proposes micro-macro laws for some typical ordered lattice configurations. In the last paragraph $(\$ 6.3)$ dedicated to the periodic random lattice network, Ostoja-Starzewski proposes numerical tests to calibrate the model. The analytical approach is limited to an ordered and homogeneous configuration. This idea is well synthesized by Potyondy and Cundall $(6, \S 3.1)$ who write:

"For continuum models, the input properties (such as modulus and strength) can be derived directly from measurements performed on laboratory specimens. For the $B P M^{1}(\ldots)$ the input properties of the components usually are not known. (...) For the general case of arbitrary packing of arbitrarily sized particles, the relation is found by means of a calibration process (... )". To summarize, for the random domains, the quantification of the microscopic parameters requires some numerical tests called a calibration procedure. This problem has been intensively studied for the cohesive dual spring model. Hentz et al. (7) have proposed numerical quasi-static uni-axial traction tests to calibrate the bond parameters in regard to the macroscopic Young's modulus and Poisson's ratio. Note that these authors have introduced an energy criterion to reduce dispersion of the macroscopic emergent properties. Then, numerical dynamic tests are used to check the dynamic properties. Fakhimi et al. (8) have used calibration curves and dimensional analysis instead of the trial and error approach. Tamarez and Plesha (9) have used analytical formulations of a 2D elementary volume (a unit cell).

The cohesive beam model was first introduced by Herrmann in 1988 (16). This model was used in a 2D ordered lattice network $(17,18)$, then in a disordered $2 \mathrm{D}$ lattice network $(11,19,20,21,22)$. In reference (11), the authors have considered that the microscopic and macroscopic Young's moduli and Poisson's ratios must be similar. The Beam dimensions (cross section and inertia momentum) are chosen thanks to a numerical recursive algorithm to satisfy an uniform elastic continuum condition. The main subject of other papers is the development of fracture models. The calibration methods are not described in depth. Researchers simply recommend using experimental and numerical tests.

In this study, unlike that of Schlangen(11), the mechanical properties of the cohesive beams will not be considered as similar to the reference material. The microscopic local properties could be driven to produce discrete matter internal reorganization under loading that induces the desired behaviors at the macroscopic scale.

In this paper the 3D cohesive beam model is briefly described in the first section. Next, a new calibration method adapted to the simulation of a perfectly homogeneous, isotropic, elastic material with 3D discrete elements bonded by beams is introduced in three steps.

1. The first step is the geometrical analysis of the initial domain configuration, presented in three parts.

\footnotetext{
1 "Bonded Particle Model", discrete element model used by Potyondy and Cundall
} 
homogeneity: Voids inside the discrete material are minimized thanks to a compaction algorithm. A method is developed to ensure the validity of the sphere packing with appropriate control criteria.

isotropy: An original and simple method to define and quantify the geometrical isotropy is presented.

fineness: A solution to define the micro-scale and macro-scale ratio (the critical number of discrete elements number that allows the emergence of stable geometrical properties) is discussed.

2. Then the influence of the beam parameters on the macroscopic behaviors is studied by quasi-static simulations. A micro-macro tendency is used to develop an original calibration method for the elastic parameters (macroscopic Young's modulus and Poisson's ratio).

3. The same methodology is used for the calibration of the mass properties, by dynamic simulations.

4. The influence of the discrete element size on the calibration results is studied. The independence of the calibrated parameters with regard to the discrete element size is a property that appears to be essential when quantitative results are wanted.

The overall method is applied to the studied material, the silica glass. The calibration method is validated through a large number of static and dynamic tests.

\section{Cohesive beam bond model}

Figure 1 shows two discrete elements bonded by a cohesive beam. The cylindrical geometry is chosen because it's dimensional description requires only two independent parameters: a length $L_{\mu}$ and a radius $r_{\mu}{ }^{2}$. The mechanical properties are also linked to the cohesive beams: a Young's modulus $E_{\mu}$ and a Poisson's ratio $v_{\mu}$. These four geometric and mechanical parameters suffice to describe the cohesive beam. Note that the cohesive beams are mass-less; mass properties are assigned only to the discrete elements.

For the sake of clarity figure 2 shows a configuration in which the discrete elements have been moved away. The cohesive beam is symbolized by its median line. Both cohesive bond ends are fixed to the discrete element centers $O_{1}$ and $O_{2}$. The discrete element frames

\footnotetext{
${ }^{2}$ To distinguish micro from macro properties, micro parameters are denoted by ' $\mu$ ' and macro parameters by ' $M$ '.
}

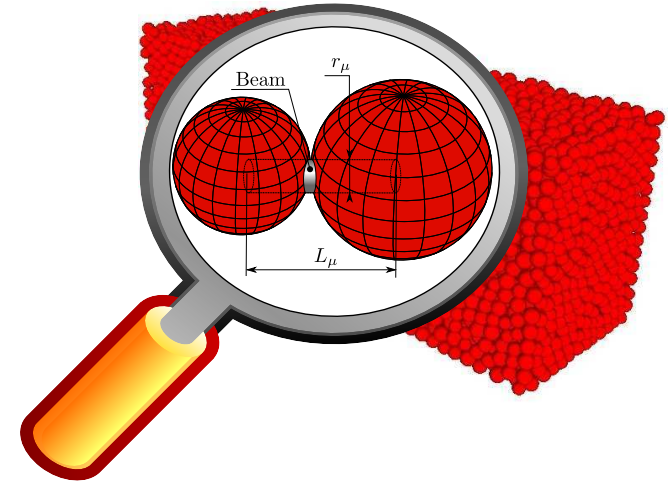

Figure 1: The cohesive beam bond

$F_{1}\left(O_{1}, \overrightarrow{X_{1}}, \overrightarrow{Y_{1}}, \overrightarrow{Z_{1}}\right)$ and $F_{2}\left(O_{2}, \overrightarrow{X_{2}}, \overrightarrow{Y_{2}}, \overrightarrow{Z_{2}}\right)$ are oriented such that $\vec{X}_{1}$ and $\overrightarrow{X_{2}}$ are normal to the beam cross section ends. At the initial time, the beams are relaxed (figure $2 \mathrm{a})$. Figure $2 \mathrm{~b}$ shows the cohesive beam in a loading state induced by the discrete element movement relatively to the initial configuration.

The analytical model of Euler-Bernoulli beam is well known (23). In reference $(24, \S 6.2)$, the author describe a stiffness matrix expressed in the beam local frame for a finite element application. Figure $2 b$ illustrates the beam local frame positioning. The center of discrete element $1\left(O_{1}\right)$ is considered as the origin. The "aligned" configuration, in which $\overrightarrow{O_{1} O_{2}}=k \overrightarrow{X_{1}}=-k \overrightarrow{X_{2}}$, is considered as the non-bending state and is taken as reference. Consequently, the cohesive beam local frame $F(O, \vec{X}, \vec{Y}, \vec{Z})$ is oriented such that (see figure $2 \mathrm{~b}$ ):

$$
\vec{X}=\frac{\overrightarrow{O_{1} O_{2}}}{\left\|\overrightarrow{O_{1} O_{2}}\right\|} \text { and } \vec{Y}=\vec{X} \wedge \overrightarrow{X_{1}} \text { and } \vec{Z}=\vec{X} \wedge \vec{Y}
$$

In the local frame $F$, the deflections at $O_{1}$ and $O_{2}$ are null. Cross section bending rotations at $O_{1}$ and $O_{2}$ are defined, respectively, by $\theta_{1}=\left(\vec{X}, \overrightarrow{X_{1}}\right)$ and $\theta_{2}=$ $\left(\overrightarrow{-X}, \overrightarrow{X_{2}}\right)$. Consequently, the force and torque reactions acting on discrete elements 1 and 2 are: 


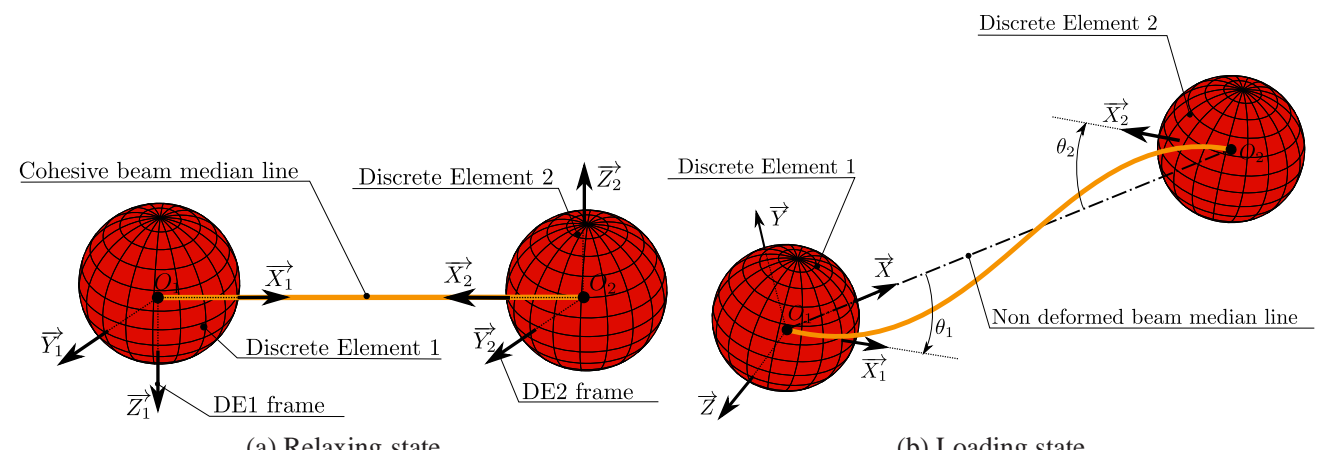

(a) Relaxing state

(b) Loading state

Figure 2: Cohesive beam bond configuration

$$
\begin{aligned}
\overrightarrow{F_{D E 1}} & =+E_{\mu} S_{\mu} \frac{\Delta l_{\mu}}{l_{\mu}} \vec{X} \\
& -\frac{6 E_{\mu} I_{\mu}}{l_{\mu}^{2}}\left(\left(\theta_{2 z}+\theta_{1 z}\right) \vec{Y}+\left(\theta_{2 y}+\theta_{1 y}\right) \vec{Z}\right) \\
\overrightarrow{F_{D E 2}} & =-E_{\mu} S_{\mu} \frac{\Delta l_{\mu}}{l_{\mu}} \vec{X} \\
& +\frac{6 E_{\mu} I_{\mu}}{l_{\mu}^{2}}\left(\left(\theta_{2 z}+\theta_{1 z}\right) \vec{Y}-\left(\theta_{2 y}+\theta_{1 y}\right) \vec{Z}\right) \\
\overrightarrow{T_{D E 1}} & =+\frac{G_{\mu} I o_{\mu}}{l_{\mu}}\left(\theta_{2 x}-\theta_{1 x}\right) \vec{X} \\
& -\frac{2 E_{\mu} I_{\mu}}{l_{\mu}}\left(\left(\theta_{2 y}+2 \theta_{1 y}\right) \vec{Y}-\left(\theta_{2 z}+2 \theta_{1 z}\right) \vec{Z}\right) \\
& -\frac{2 E_{\mu} I_{\mu}}{l_{\mu}}\left(\left(2 \theta_{2 y}+\theta_{1 y}\right) \vec{Y}-\left(2 \theta_{2 z}+\theta_{1 z}\right) \vec{Z}\right) \\
T_{D E 2} & =-\frac{G_{\mu} I o_{\mu}}{l_{\mu}}\left(\theta_{2 x}-\theta_{1 x}\right) \vec{X}
\end{aligned}
$$

where:

- $\overrightarrow{F_{D E 1}}$ and $\overrightarrow{F_{D E 2}}$ are the beam force reactions acting on discrete elements 1 and 2 .

- $\overrightarrow{T_{D E 1}}$ and $\overrightarrow{T_{D E 2}}$ are the beam torque reactions acting on discrete elements 1 and 2.

- $l_{\mu}$ and $\Delta l_{\mu}$ are the initial beam length and the longitudinal extension.

- $\overrightarrow{\theta_{1}}\left(\theta_{1 x}, \theta_{1 y}, \theta_{1 z}\right)$ and $\overrightarrow{\theta_{2}}\left(\theta_{2 x}, \theta_{2 y}, \theta_{2 z}\right)$ are the rotations of beam cross section at the points $O_{1}$ and $O_{2}$ expressed in the beam local frame.

- $S_{\mu}, I o_{\mu}$ and $I_{\mu}$ are the beam cross section area, polar moment of inertia and moment of inertia along $\vec{Y}$ and $\vec{Z}$.

- $E_{\mu}$ and $G_{\mu}$ are the Young and shear modulus.

Note that reaction force and torque are expressed in the beam local frame $F(O, \vec{X}, \vec{Y}, \vec{Z})$. The four parameters that define the micro beam, $L_{\mu}, r_{\mu}, E_{\mu}$ and $v_{\mu}$ have only an influence on the elastic behavior of the assembly.

\section{Explicit dynamic algorithm}

The numerical resolution is based on an explicit integration scheme well adapted to massive DEM simulation (25) and high velocity phenomena such as fracturing or impact simulation. Many explicit schemes can be used : the Verlet velocity, Runge-Kutta, leapfrog or gear's method...(26, §13). In reference $(25)$, the authors have compared these algorithms "in terms of accuracy, stability and CPU efficiency". It appears that all of them give approximately the same efficiency.

Velocity Verlet scheme is chosen for its simplicity . Discrete element position and velocity are estimated by:

$$
\begin{aligned}
& \vec{p}(t+d t)=\vec{p}(t)+d t \overrightarrow{\dot{p}}(t)+\frac{d t^{2}}{2} \vec{p}(t) \\
& \vec{p}(t+d t)=\overrightarrow{\dot{p}}(t)+\frac{d t}{2}(\vec{p}(t)+\vec{p}(t+d t))
\end{aligned}
$$

where :

- $t$ is the current time and $d t$ is the integration time step. 
- $p(t), \dot{p}(t)$ and $\ddot{p}(t)$ is the discrete element linear position, velocity and acceleration.

The discrete element orientations are described by quaternions, noted $q(t)$, that allow an efficiency way to compute the rotation of the local frames associated with discrete elements $(27, \S 2.5)$. Quaternion is linked to the angular velocity with the following equality $(26, \S 10.5)$ :

$$
\dot{q}(t)=\frac{1}{2} \vec{\omega}(t) q(t)
$$

Where $\vec{\omega}(t)$ is the angular velocity of a discrete element. The velocity Verlet scheme is also applied to quaternion $q(t)$, with:

$$
\begin{aligned}
& q(t+d t)=q(t)+d t \dot{q}(t)+\frac{d t^{2}}{2} \ddot{q}(t) \\
& \dot{q}(t+d t)=\dot{q}(t)+\frac{d t}{2}(\ddot{q}(t)+\ddot{q}(t+d t))
\end{aligned}
$$

To prevent $q(t)$ numerical drift, the quaternion must be normalized at each time step. Algorithm 1 details the encapsulation of Verlet velocity in an explicit dynamic resolution. Note that this numerical scheme is not welladapted to quasi-static simulation. Special care, described later in this paper, will be taken with this kind of test.

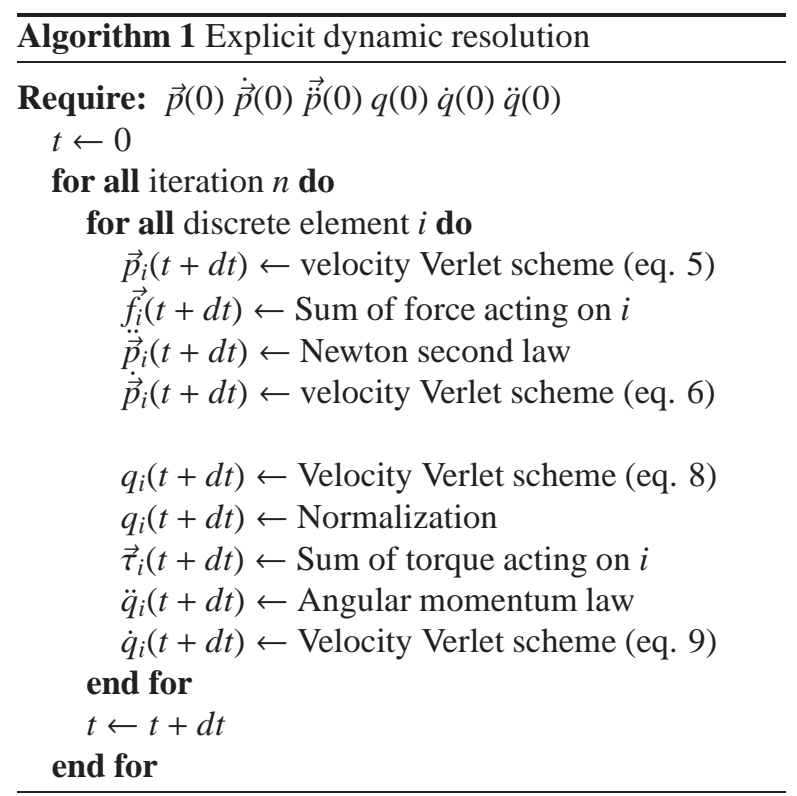

\section{Geometrical properties of the initial discrete do- main}

The initial discrete domain geometry must be in accordance with the structural properties of the simulated material. In the case of the perfectly elastic solid, the main properties are homogeneity and isotropy. The geometrical discrete domain configuration impacts the mechanical behavior at the macroscopic scale. In ref. $(11,12)$ the authors have observed that ordered arrangements promote anisotropic phenoma such as preferred crack paths or the non-uniform propagation of elastic waves.

In the case of the spherical discrete elements, artificial voids are generated between discrete elements inside the material. At this scale, the discrete material is not homogeneous. To reduce undesirable voids, the discrete domain must be initially compacted.

This subsection first discusses a method that ensures and verifies the geometrical homogeneity and isotropy. Then, the influence of the discrete domain refining is studied.

\subsection{Homogeneity}

To decrease the artificial voids between the discrete elements, various packing processes can be used, for example, dynamic methods such as iterative growth algorithm (28) or isotropic compression (29) or geometric algorithm (30). In this study, the discrete domain is generated following a dynamic custom recipe. The figure 3 shows the initial configuration. The discrete elements are placed along a plane-parallel grid. an uniform dispersion law, detailed later in this section, is applied to discrete element radii. The compaction simulation consist of :

- applying an horizontal sinusoidal movement to the "Shear Wall" set.

- applying a vertical pressure to "Pressure Wall" set.

- confining the discrete domain in a given volume thanks to the "Repulsive Walls" which apply an elastic repulsion law.

For an identical or small dispersion radius, the sphere packing should be similar to a "Random Close Packing" (RCP) as described in (31). A first step to validate the initial discrete domain is to check its conformity with RCP definition. The domain is considered as correctly compacted, if the sphere packing gives a volume fraction value around 0.636 (32) and a cardinal number ${ }^{3}$ value around 6 (33). However, these two values do not ensure that the packing is geometrically isotropic.

\footnotetext{
${ }^{3}$ Average number of contact per discrete element.
} 


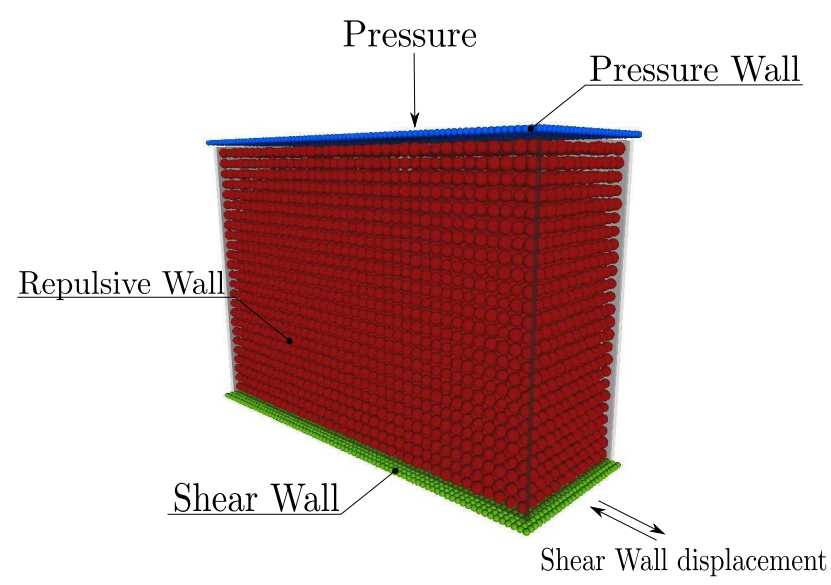

Figure 3: Shear compaction method

\subsection{Isotropy}

The definition of geometrical isotropy must be clarified before proposing a criterion. Cambou (34, Introduction, §3.6) defines the geometric anisotropy as the distribution of contact directions. If this distribution is perfectly homogeneous, the domain is considered as geometrically isotropic. To summarize, an assertion could be formulated as: the geometrical isotropy is a necessary condition to ensure the mechanical isotropy of the simulated material. To "measure" the geometrical organization of granular material, the authors in $(35, \S 1.2 .2)$ have exploited a mathematical tool called the "fabric tensor". However, this tool cannot be used to determine the isotropy in a simple way (36). A more intuitive method based on a simple geometric computation and statistical analysis is proposed. It is an extension to $3 \mathrm{D}$ space of classical 2D graphs that classify contact into a direction subset (for example references (36) and (20)).

Contacts are grouped into a subset depending on their orientation in 3D space. All the members in a set have a quite similar spatial orientation. To group contacts in orientation sets, a platonic solid (a "geode") of 320 equal faces is built. Each contact is placed at the geode center, and added to the corresponding intersected face group (see figure 4). The result is a kind of 3D histogram in which each class maps an orientation subset (a sort of discretized solid angle); any class weight gives the density of contact orientations that matches the solid angle. For a low dispersion of class weights, the discrete domain is considered as geometrically isotropic (an homogeneous distribution of contact orientation in 3D space).

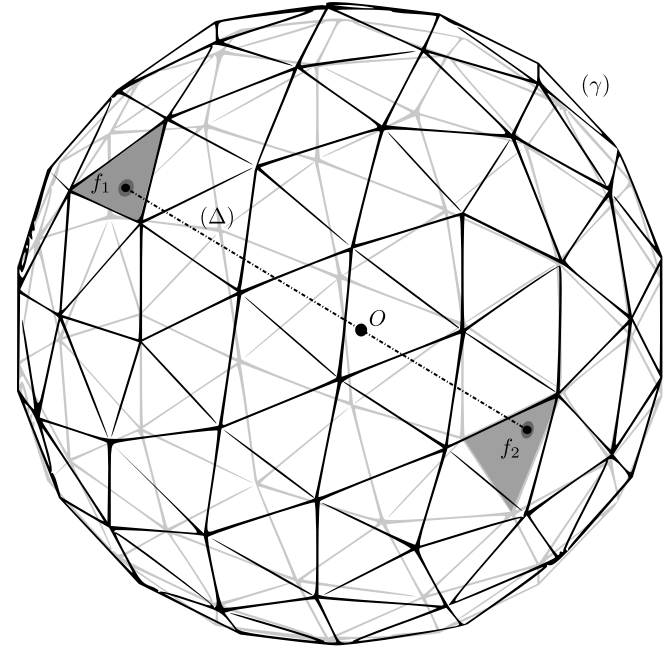

Figure 4: Platonic solid (Geode) used as a reference geometry to classify the contact orientation

To illustrate this method, the influence of the particle size distribution on isotropy is studied. It is known from literature (31) that to prevent an ordered packing configuration, a dispersion (noted $\kappa$ ) must be applied on discrete elements radius. In this study, the dispersion $\kappa$ is uniform and is defined as the dispersion range :

$$
\kappa=\frac{R_{\max }-R_{\min }}{\bar{R}}
$$

where $R_{\max }, R_{\min }$ and $\bar{R}$ are the maximum, minimum and average discrete element radius values.

Figures 5 shows the geometrical arrangement for two values of the dispersion parameter $\kappa$. The figure illustrates the influence of radius dispersion on the geometrical arrangement (figures $5 \mathrm{a}$ and $5 \mathrm{~b}$ ) and on the contact orientation (figures $5 \mathrm{c}$ and $5 \mathrm{~d}$ ). For a distribution range $\kappa=0 \%$ the packing seems to be perfectly ordered. The perfect arrangement is strongly anisotropic. In contrast, a higher dispersion value $(\kappa=25 \%)$ seems to promote the isotropy.

Figures $6 \mathrm{a}$ and $6 \mathrm{~b}$ show the 3D histograms used to qualify the observed level of isotropy. From these figures, it is clear that the radius dispersion value $\kappa$ highly influences the isotropy level. To quantify this level, it is proposed to compute the mean square difference between observed frequencies in geode cells $\left(f_{i}\right)$ and the uniform frequency $(1 / N)$ :

$$
e=\sum_{i=1}^{N} \frac{\left(f_{i}-\frac{1}{N}\right)^{2}}{N}
$$




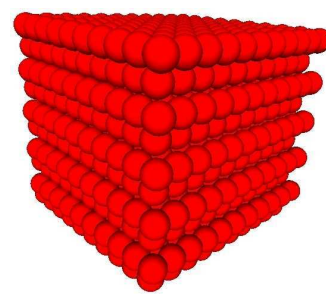

(a) Domain for $\kappa=0 \%$ (discrete element view)

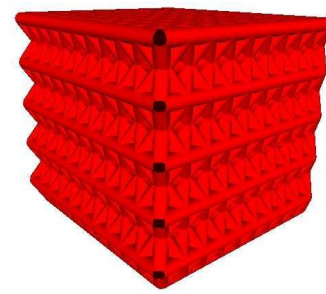

(c) Domain for $\kappa=0 \%$ (contact view)

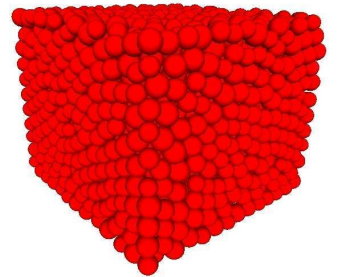

(b) Domain for $\kappa=25 \%$ (discrete element view)

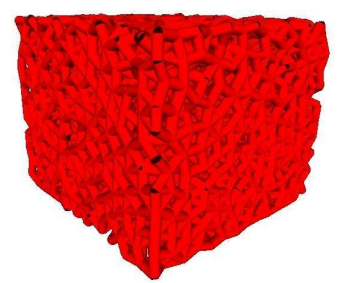

(d) Domain for $\kappa=25 \%$ (contact view)
Figure 5: Geometrical arrangement of a 3D sphere packing with different values of $\kappa$

\section{Where :}

- $N$ is the total number of cells, i.e, the 320 "geode" faces.

- $f_{i}$ is the observed frequency of the cell $i$, i.e, the ratio between the number of contact that matches the solid angle $i$ and the total contact number.

The important aspect of this criterion is the asymptotic behavior (see figure 7). Increasing radius dispersion value $\kappa$ gives an asymptotic constant limit, beginning from a $\kappa$ value of $15 \%$. This result is in accordance with the observation of Luding (37, chapter 5): "crystallization (...) does not occur for polydisperse packing with $\omega_{0} \gtrsim 0.15^{\prime \prime}$. In other words, for a dispersion value higher than $15 \%$, an ordered geometrical arrangement does not occur within the sphere packing. Thus, a value of $\kappa=25 \%$ is chosen to ensure minimal anisotropy.

\subsection{Discrete domain refining}

The three criteria that drive the domain homogeneity and isotropy are the volume fraction, the cardinal number and the mean square difference of the contact orientation packet.

This section deals with two questions:

- Do the three criteria converge if the number of discrete element per unit of volume increases?

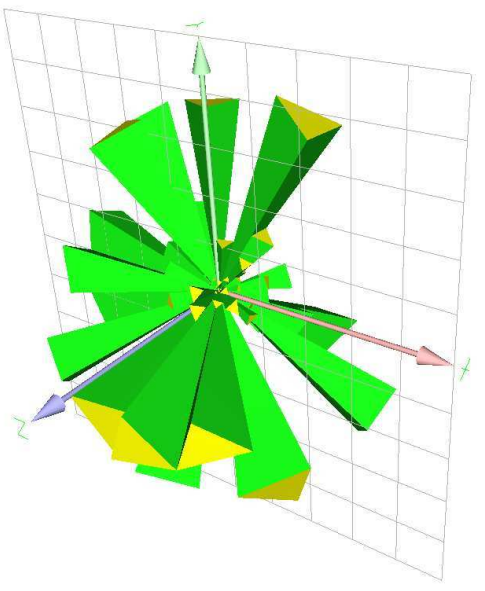

(a) $\kappa=0 \%$

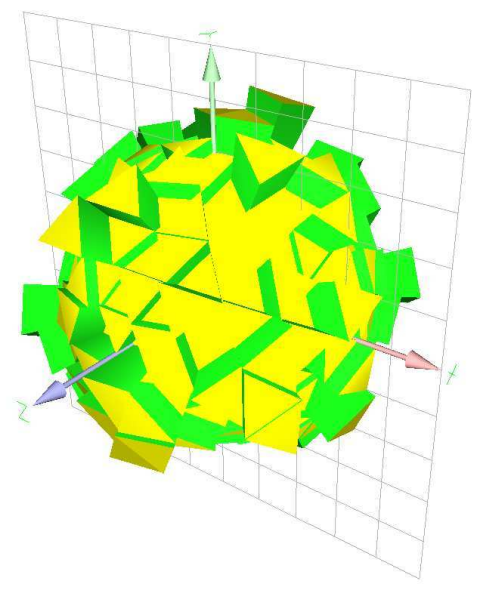

(b) $\kappa=25 \%$

Figure 6: 3D histograms of the orientation set

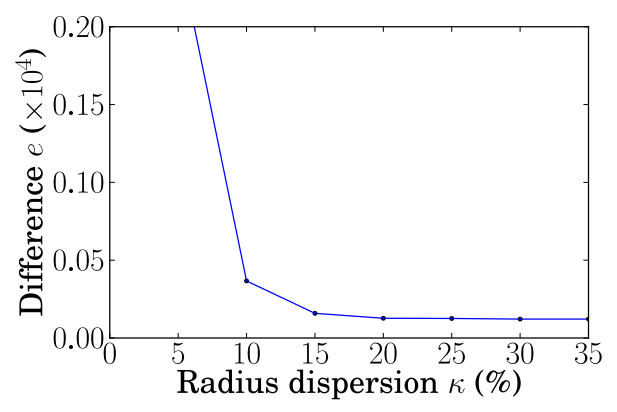

Figure 7: Evolution of the mean square difference $e$ parameter of the sampling distribution of the contact orientation packet versus radius dispersion $\kappa$ 
- In this case, is it possible to define the right number of discrete elements that allows the simulation of an homogeneous and isotropic medium?

First, the meaning of refining must be clarified. Refining consists of increasing the number of discrete elements per unit of volume. To study the influence of refining on the three criteria, 22 packing domains of identical bounding volume ${ }^{4}$ were built. To permit a statistical processing, 5 packing domains are built for each discrete element number value. Therefore, a total of 110 packing domains were analyzed.

Figures $8 \mathrm{a}, 8 \mathrm{~b}$ and $8 \mathrm{c}$ show the influence of refining on the volume fraction, cardinal number and isotropy. These values are extracted from the discrete domains built with a dispersion radius of $\kappa=25 \%$ (corresponding to the conclusion of the previous section ). Small differences could be accepted. The criteria could be classified by order of importance :

1. Isotropy is considered as the most important. This criterion highly influences the discrete sample mechanical behavior.

2. The cardinal number and the volumic fraction are less important. They allow to check the validity of the compact domain. Small differences between the sample values and the RCP values established in the literature are accepted.

The cardinal number converges to a limit value close to 6.2. For Volume fraction and isotropy slight variations are still observed for high number of discrete elements.

The discrete element number increases with refining. A high refining level brings down the computational performances. Therefore, a compromise must be made between performance and precision. For the next section, a number of 10000 discrete elements is considered as sufficient for an acceptable level of precision. For this value, the geometrical anisotropy criterion is lower than 0.0032 , the coordination number is higher than 6.2 and the volume fraction around 0.635. 10000 discrete elements in a $3 \mathrm{D}$ square domain gives the ratio between the micro-scale and the macro-scale to obtain isotropy and homogeneity at the macroscale; this ratio is around $10000^{1 / 3} \approx 21.5$.

\subsection{Conclusion}

The method presented in this section shows how to verify the initial compacted domain against the geometrical criteria. The geometrical criteria do not depend on

\footnotetext{
${ }^{4}$ Bounding volume is defined as the volume of the box of minimal size containing the discrete domain.
}

the discrete element mechanical interaction laws. The presented method can be applied to various discrete element models and applications.

An original method has been presented to quantify the isotropy of a discrete element set. This method is based on the classification of bond directions by using a platonic solid (geode).

Finally, a ratio of "macroscale over microscale" about 20 has been chosen, from the convergence curves, that control the geometrical properties of the discrete element set.

\section{Elastic calibration}

The previous section introduced a methodology to build an initial compact discrete domain that ensures an adequate, homogeneous, isotropic and geometric organization. Then, cohesive beams (see section 2) are introduced by creating a beam at each contact between two discrete elements. In further simulations, the contacts are not taken in account; only cohesive beam interactions between discrete elements are considered.

To study the influence of cohesive beam bond parameters on macroscopic elastic behavior, a parametric study using numerical quasi-static uni-axial tensile test is used.

\subsection{Quasi-static tensile test description}

A perfectly homogeneous, isotropic, elastic material is characterized by the Young's modulus and the Poisson's ratio. For real materials, these parameters are generally determined by quasi-static tensile tests. These experimental procedures can be also applied to a numerical sample.

\subsubsection{From discrete to continuous geometry}

To compute the macroscopic Young's modulus and Poisson's ratio, a perfect 3D continuous geometry is associated to the compact discrete domain. This perfect geometry is the bounding shape of the compacted discrete domain. The discrete elements belonging to the domain boundaries are marked to compute the perfect geometry dimensions. With the cylinder shape, three discrete element set are marked (see figure 9):

- the "xMax" and "xMin" set are associated to faces with normal $\vec{X}$ and $\overrightarrow{-X}$.

- the "radius" set is associated with the cylinder circumference. 


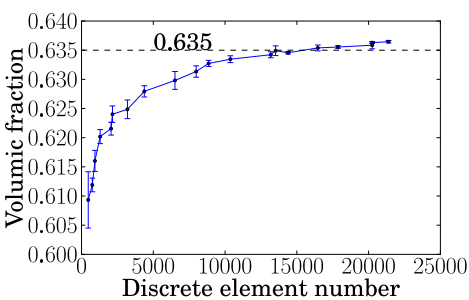

(a) Volume fraction

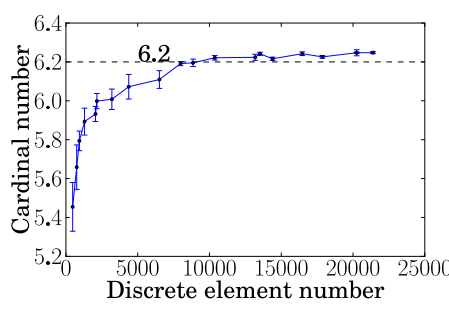

(b) Cardinal number

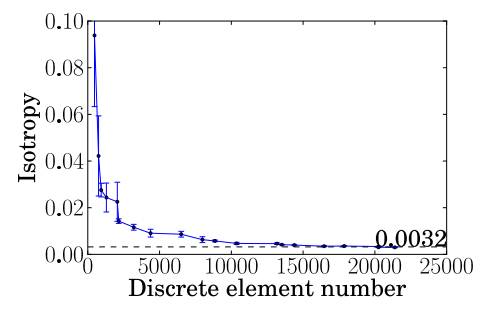

(c) Isotropy

Figure 8: Evolution of 3 geometrical criteria versus refining (for radius dispersion $\kappa=25 \%$ )

The Perfect cylinder dimensions are computed as :

$$
\begin{aligned}
& L_{M}=2 \bar{R}+\left(\frac{1}{N_{x M a x}} \sum_{i=1}^{N_{x M a x}} \overrightarrow{O G_{i}}+\frac{1}{N_{x M i n}} \sum_{i=0}^{N_{x M i n}} \overrightarrow{O G_{i}}\right) \cdot \vec{X} \\
& R_{M}=\bar{R}+\frac{1}{N_{\text {radius }}} \sum_{i=1}^{N_{\text {radius }}} \sqrt{\left(\overrightarrow{O G_{i}} \cdot \vec{Y}\right)^{2}+\left(\overrightarrow{O G_{i}} \cdot \vec{Z}\right)^{2}}
\end{aligned}
$$

where:

- $L_{M}$ and $R_{M}$ are the perfect cylinder length and radius.

- $N_{x \operatorname{Max}}, N_{x M i n}$ and $N_{\text {radius }}$ are the number of discrete elements belonging to "xMax", "xMin" and "radius" sets.

- $\overrightarrow{O G_{i}}$ is the position of the discrete element gravity center.

- $\bar{R}$ is the average discrete element radius over the entire domain.

\subsubsection{Loading}

To ensure a quasi-static tensile test, the loading force acting on the discrete element set "xMin" and "xMax", are progressively applied (linear ramp) and stabilized. The sum of forces acting on "xMax" and "xMin" are denoted by $\overrightarrow{F_{x M a x}}$ and $\overrightarrow{F_{x M i n}}$. These two forces, acting along $\vec{X}$ for $\overrightarrow{F_{x M a x}}$ and $\overrightarrow{-X}$ for $\overrightarrow{F_{x M i n}}$, have equal norms and are opposed. To check the quasi-static properties, the kinetic and deformation energies are computed and stored during the numerical test. Figure 10 confirms that a progressive loading gives a negligible kinetic energy and ensures a quasi-static aspect of the simulation.

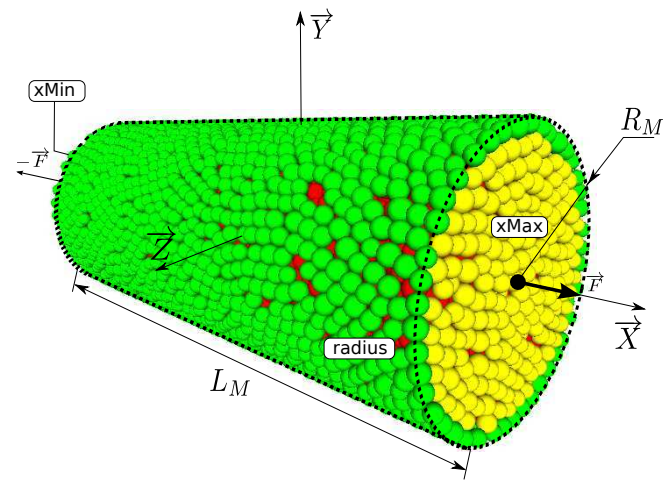

Figure 9: Perfect cylinder associated with a discrete domain with $\kappa=25 \%$

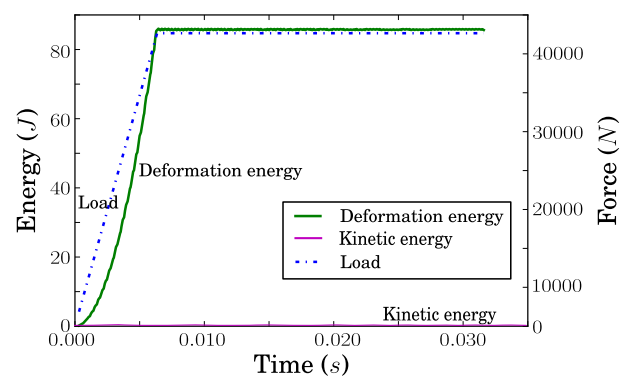

Figure 10: Kinetic and deformation energy during a quasi-static tensile test (computed with a time step $\Delta t=$ $3.10^{-7} s$ and $a$ number of iteration it $=100000$ ) 


\subsubsection{Young's modulus and Poisson's ratio computa- tion}

The Young's modulus and the Poisson's ratio can be easily determined for the cylinder sample by using the material strength analytical formulations:

$$
\begin{aligned}
E_{M} & =\frac{F / S_{M_{o}}}{\Delta L_{M} / L_{M_{o}}} \\
v_{M} & =-\frac{\Delta R_{M} / R_{M_{o}}}{\Delta L_{M} / L_{M_{o}}}
\end{aligned}
$$

where:

- $L_{M_{o}}, R_{M_{o}}$ and $S_{M_{o}}$ are the initial bounding cylinder dimensions (respectively: length, radius, and section).

- $E_{M}$ and $v_{M}$ are the macroscopic Young modulus and Poisson ratio.

- $F$ is the normal force.

The explicit numeric schemes are not well-adapted to the quasi-static simulation. The system vibrates around the static solution. To allow a convergence, a pure numerical damping factor is introduced in the numerical scheme as described by Tchamwa and Wielgosz (38). This is a decentered explicit integration scheme that allow high frequency dissipation. This scheme is very similar to the Velocity Verlet algorithm. The dissipation is controlled with a single parameter $\varphi$. Only the second time derivative equality is modified. The equation 6 and 9 become :

$$
\begin{aligned}
& \vec{p}(t+d t)=\dot{\vec{p}}(t)+\varphi \frac{d t}{2}(\vec{p}(t)+\vec{p}(t+d t)) \\
& \dot{q}(t+d t)=\dot{q}(t)+\varphi \frac{d t}{2}(\ddot{q}(t)+\ddot{q}(t+d t))
\end{aligned}
$$

The value $\varphi=1.3$ is used to allow a high convergence rate to the static solution.

\subsection{Parametric study}

The cohesive beam bond is defined by four parameters:

- two geometrical parameters: length $L_{\mu}$ and radius $r_{\mu}$.

- two mechanical parameters: Young's modulus $E_{\mu}$ and Poisson's ratio $v_{\mu}$.
The value of the cohesive beam bond length depends on the distance between discrete element centers and is not a free parameter. The three others parameters are free and must be quantified.

The adimensional cohesive beam radius parameter noted $\tilde{r_{\mu}}$ will be preferred to the beam radius. It allows a definition that does not depend on the discrete element sizes. It is defined as the ratio between the cohesive beam radius and the average discrete element radius $\bar{R}$. Note that this value is the same for all the cohesive beams; consequently, all the cohesive beam radii are equal.

\subsubsection{Microscopic Poisson's ratio influence}

Figure 11 shows the evolution of the macroscopic Young's modulus $E_{M}$ and Poisson's ratio $v_{M}$ for the different microscopic Poisson's ratio $v_{\mu}$ values in the range $[0,1 / 2]$. It is observed that the microscopic Poisson's ration $v_{\mu}$ does not influence the macroscopic Young's modulus $E_{M}$ and the macroscopic Poisson's ratio $v_{M}$ significantly.

Equations 3 and 4 show that the microscopic shear modulus $G_{\mu}$, and consequently the microscopic Poisson's ratio $v_{\mu}$ plays a role in local torsion loading (in a cohesive beam). During a quasi-static tensile test, the contribution of the local torsion energy is minor (see figure 12). Each energy represented on this figure are the sum of the local elastic energy stored by the cohesive beams. The total elastic energy is split up into :

Tension energy characterized by the sum of the cohesive beam works of the normal forces.

Bending energy characterized by the sum of the cohesive beam works of the bending torques.

Torsion energy characterized by the sum of the cohesive beam works of the torsion torques.

To summarize, the influence of the microscopic Poisson's ratio is negligible. Consequently, the microscopic Poisson's ratio values can be chosen arbitrarily. For the rest of the study a value of 0.3 is chosen.

\subsubsection{Microscopic Young's modulus influence}

Figure 13 shows the evolution of the macroscopic parameters as a function of microscopic Young modulus for different values of the microscopic radius ratio. The next table gives an outline of these evolutions.

\begin{tabular}{|c|c|c|}
\hline $\begin{array}{l}\text { Macroscopic } \\
\text { parameters }\end{array}$ & $\begin{array}{c}\text { Macroscopic Young's } \\
\text { modulus } E_{M}\end{array}$ & $\begin{array}{c}\text { Macroscopic Poisson's } \\
\text { ratio } v_{M}\end{array}$ \\
\hline $\begin{array}{l}\text { Functions } \\
\text { Evolution }\end{array}$ & $E_{M}=f_{1}\left(E_{\mu}\right)$ & $v_{M}=f_{2}\left(E_{\mu}\right)$ \\
increasing linear & constant function \\
Figures & function & $13 \mathrm{~b}$ \\
\hline
\end{tabular}




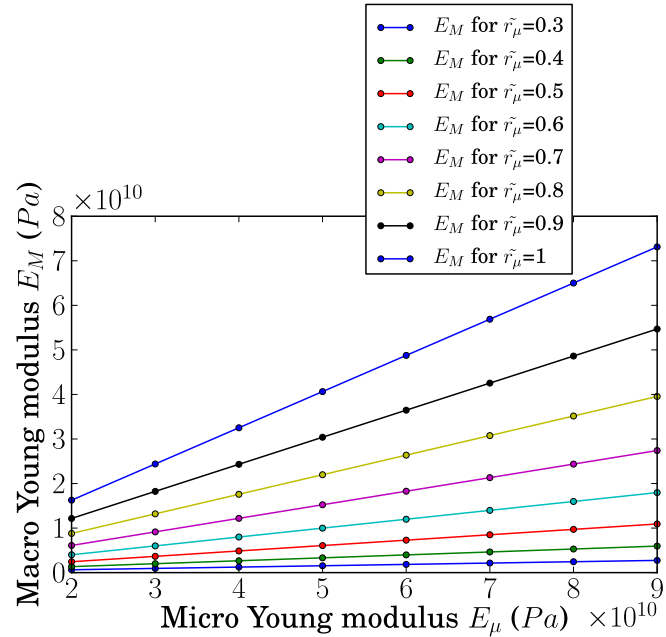

(a) $E_{M}$ evolution as a function of $E_{\mu}$

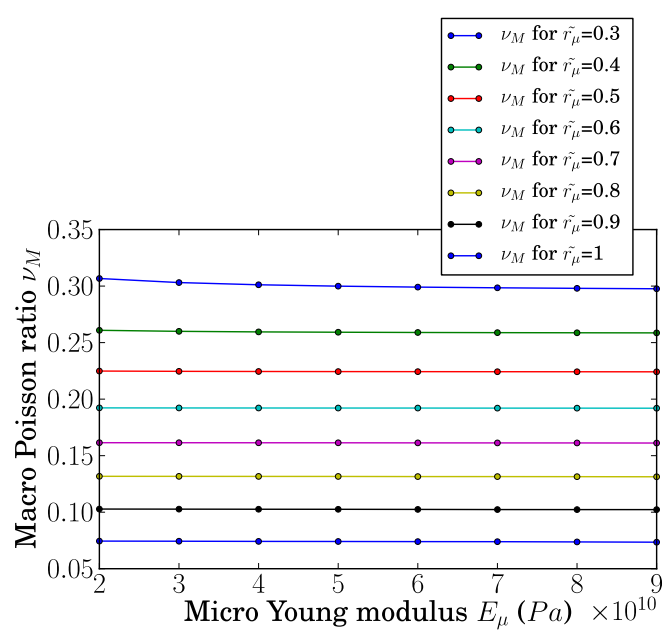

(b) $v_{M}$ evolution as a function of $E_{\mu}$

Figure 13: Microscopic Young's modulus $E_{\mu}$ influence on the macroscopic parameters $E_{M}$ and $v_{M}$

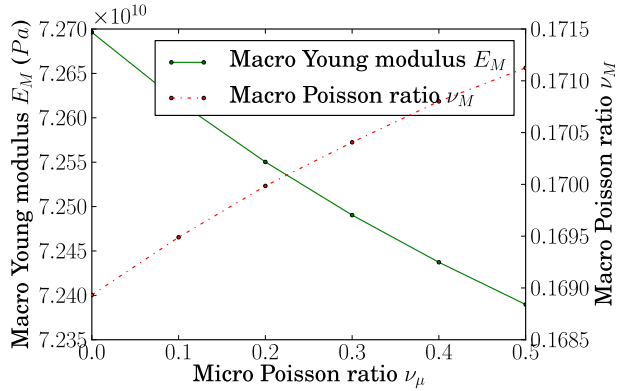

Figure 11: Influence of $v_{\mu}$ on $E_{M}$ and $v_{M}$

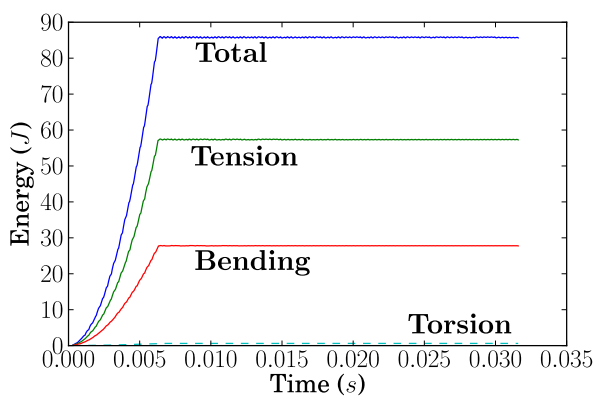

Figure 12: Energy breakdown of total elastic energy stored by cohesive beams for the quasi-static tensile test (computed with a time step $\Delta t=3 \cdot 10^{-7} s$ and a number of iteration it $=100000$ )

\subsubsection{Microscopic radius ratio influence}

Figure 14 shows the evolution of the macroscopic parameters as a function of the microscopic radius ratio $\tilde{r_{\mu}}$ for different values of the microscopic Young's modulus. The next table gives an outline of these evolutions.

\begin{tabular}{|c|c|c|}
\hline $\begin{array}{l}\text { Macroscopic } \\
\text { parameters }\end{array}$ & $\begin{array}{c}\text { Macroscopic Young's } \\
\text { modulus } E_{M}\end{array}$ & $\begin{array}{c}\text { Macroscopic Poisson's } \\
\text { ratio } v_{M}\end{array}$ \\
\hline Functions & $E_{M}=f_{3}\left(\tilde{r_{\mu}}\right)$ & $v_{M}=f_{2}\left(\tilde{r_{\mu}}\right)$ \\
Evolution & increasing quadratic & decreasing quadratic \\
& function. & function \\
Figures & $14 \mathrm{a}$ & $14 \mathrm{~b}$ \\
\hline
\end{tabular}

\subsection{Calibration method}

Section 5.2 has described the influences of the microscopic parameters $v_{\mu}, E_{\mu}$ and $\tilde{r_{\mu}}$ on the macroscopic parameters $E_{M}$ and $v_{M}$. These influences will be used to develop a calibration methodology in two steps: the calibration of microscopic radius ratio, then the calibration of microscopic Young's modulus. Section 5.2.1 has shown that the influence of the microscopic Poisson's ratio $v_{\mu}$ is negligible. Its value is arbitrarily fixed at 0.3 .

\subsubsection{Microscopic radius ratio $\tilde{r_{\mu}}$ calibration}

Section 5.2.2 have shown that the influence of the microscopic Young's modulus $E_{\mu}$ is very small on macroscopic Poisson ratio $v_{M}$ (figure 13b).

The first calibration step considers that the macroscopic Poisson's ratio $v_{M}$ does not depend on the microscopic Young's modulus $E_{\mu}$. A single macroscopic Poisson's ratio $v_{M}$ value is associated with each microscopic radius ratio $\tilde{r_{\mu}}$. The figure 15 shows the evolution of the macroscopic Poisson's ratio $v_{M}$ as a function of 


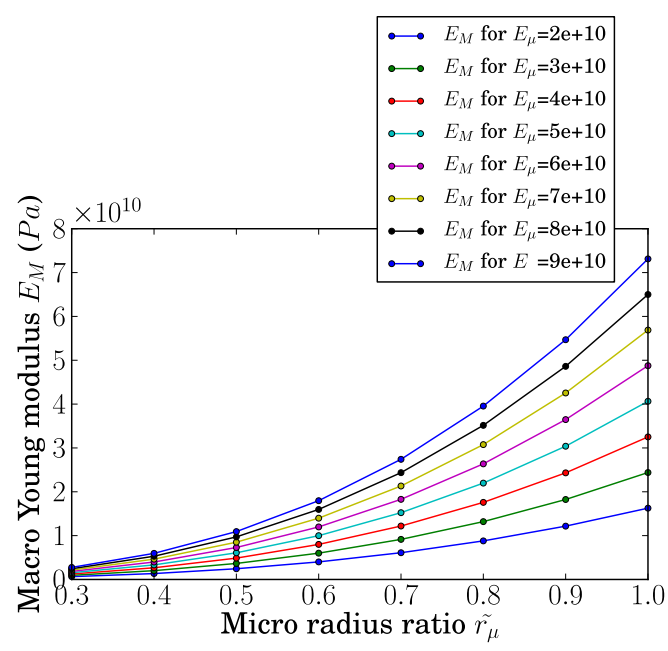

(a) $E_{M}$ evolution as a function of $\tilde{r_{\mu}}$

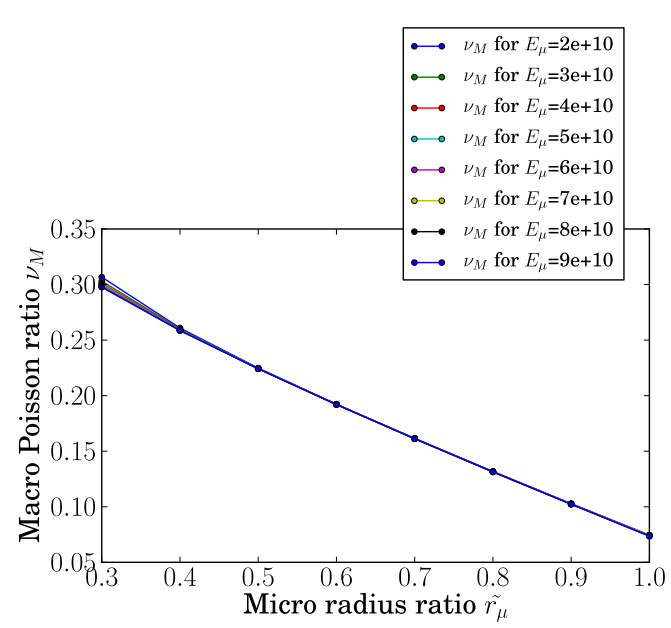

(b) $v_{M}$ evolution as a function of $\tilde{r_{\mu}}$

Figure 14: Microscopic radius ratio $\tilde{r_{\mu}}$ influence on the macroscopic parameters $E_{M}$ and $v_{M}$

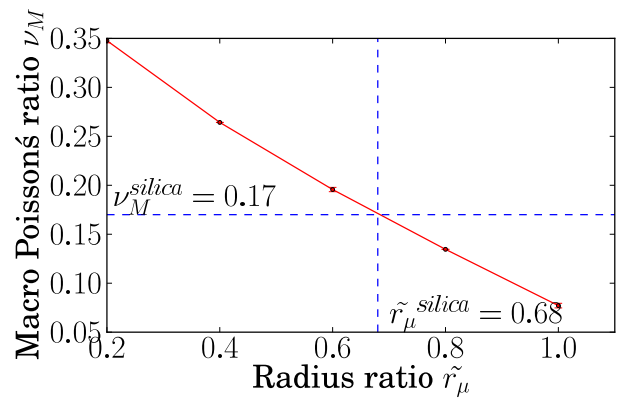

Figure 15: Radius ratio $\tilde{r_{\mu}}$ calibration for fused silica

the microscopic radius ratio $\tilde{r_{\mu}}$. This description allows the deduction of a microscopic radius ratio $\tilde{r_{\mu}}$ value corresponding to a desired-value of the macroscopic Poisson's ratio $v_{M}$. Figure 15 shows an application for the silica glass material. In this case, a microscopic radius ratio value $o f \tilde{r_{\mu}}$ silica $\approx 0.71$ is found to correspond to the silica Poisson's ratio value $v_{M}^{\text {silica }} \approx 0.17$.

\subsubsection{Microscopic Young modulus $E_{\mu}$ calibration}

Figure 16 shows the evolution of the macroscopic Young's modulus $E_{M}$ as a function of the microscopic Young's modulus $E_{\mu}$ for a radius ratio (evaluated in the previous section) of ${\tilde{r_{\mu}}}^{\text {silica }}=0.71$. This evolution allows the deduction of a microscopic Young's modulus value for a desired-value of the macroscopic Young's modulus. In the case of silica glass, the microscopic Young's modulus $E_{\mu}^{\text {silica }} \approx 265 \mathrm{GPa}$ is found to corre-

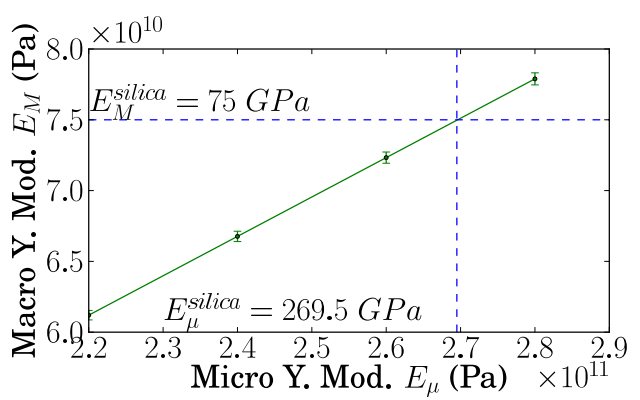

Figure 16: Microscopic Young modulus $E_{\mu}$ calibration of fused silica

spond to the silica glass Young's modulus value $E_{M}^{\text {silica }} \approx$ $72.5 \mathrm{GPa}$ (see figure 16).

\subsection{Study of assembly dependency}

To apply the micro beam model to any material geometry, it must be verified that the calibration results do not depend on the number of discrete elements in a given material volume. To check this property, many discrete samples (with similar bounding dimensions) were built with an increasing number of discrete element (see figures 18). The samples satisfy the criteria established in section 4. To take into account the variability of the sample geometry, four different samples were built with the same discrete element number. The figure 17 shows the evolution of the macroscopic pa- 


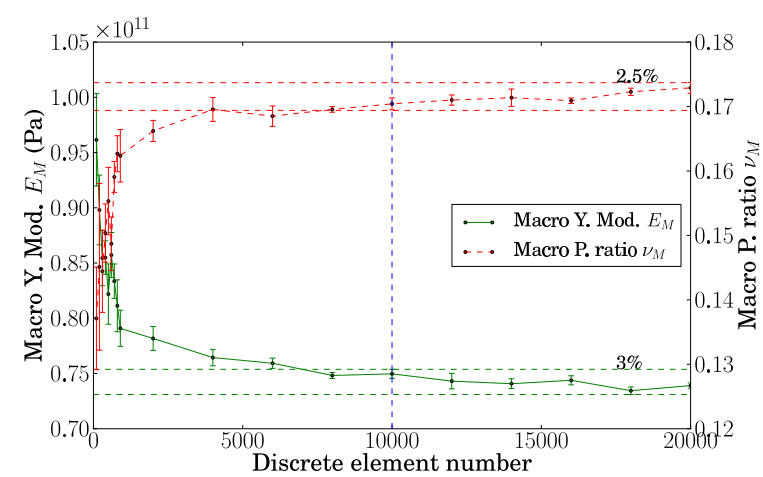

Figure 17: Evolution of the macroscopic parameters $E_{M}$ and $v_{M}$ as a function of the discrete element number.

rameters $E_{M}$ and $v_{M}$ as a function of the discrete element number.

It appears that for a number of discrete element over 7 500, the macroscopic Young modulus $E_{M}$ fluctuates around $7 \%$ and the macroscopic Poisson ratio around $v_{M} 5 \%$

Hentz (7) shown that the Liao calibration methodology for the dual spring model (39) gives a dispersion around $28 \%$ for the Young modulus and $16 \%$ for the Poisson's ratio. To improve the accuracy, Hentz has introduced an energy criterion to reduce the dispersion around $12 \%$ and $10 \%$. But this criterion is assembly dependent and must be computed for each sample.

The beam cohesive model associated to the compaction criteria presented in section 4 allow a better precision without any re-computation.

\subsection{Validation tests}

The previous subsections show a methodology to calibrate the microscopic parameters from the macroscopic elastic parameters values. Tables 1 and 2 summarize the results obtained for the silica glass material.

These microscopic elastic parameters are used to build a cylindrical numeric sample of silica glass. This sample is submitted to quasi-static tensile, bending and torsion testing, in which the "xMin" discrete element set is fixed and "xMax" set is loaded. To reproduce a quasistatic aspect, the loads are applied gradually (cf section 5.1.2)

Free face displacement and rotation given by the numerical simulations are compared to the results given by the strength of material theory. Table 3 summarizes the differences as a percent between numerical and theoretical results. The higher difference is obtained for

\begin{tabular}{|c|c|}
\hline Young's Modulus & Poisson's ratio \\
\hline \hline$E_{M}=72.5 \mathrm{GPa}$ & $v_{M}=0.17$ \\
\hline
\end{tabular}

Table 1: Macroscopic silica glass elastic values

\begin{tabular}{|c|c|c|}
\hline Young's Modulus & Poisson's ratio & Radius ratio \\
\hline \hline$E_{\mu}=265 \mathrm{GPa}$ & $v_{\mu}=0.3$ & $\tilde{r_{\mu}}=0.71$ \\
\hline
\end{tabular}

Table 2: Microscopic silica glass elastic values

the torsion test and is less than seven percent. These errors are considered acceptable. The causes are multiple and are inherent to the random discrete element positioning: imperfect loading and imprecise measurement of boundary geometry.

\section{Dynamic calibration}

The previous section deals with the elastic calibration. This method allows to the calibration of the three microscopic elastic parameters (Young's modulus, radius and Poisson's ratio) to obtain the elastic behavior at the macroscopic scale. To quantitatively simulate dynamic phenomena likes cracks or impacts, it is also necessary to calibrate the microscopic mass parameters.

The discrete elements mass parameters (inertia matrix and masses) depend on the discrete element volume and density. The discrete element geometries are initially determined. Therefore, only the discrete element density can be adjusted. In the same way as the elastic parameters, the microscopic density can be different from the macroscopic density to correct the voids between the discrete elements in a compacted domain. Therefore, the density will be considered as the only free parameter.

A very simple calibration criterion is chosen. This criterion ensures mass equality between the discrete and continuous domains:

$$
\rho_{\mu}=\frac{\rho_{M} V_{M}}{\sum_{i=1}^{N} V_{\mu_{i}}}
$$

where:

- $\rho_{\mu}$ and $V_{\mu_{i}}$ are the discrete element density and volume.

- $\rho_{M}$ and $V_{M}$ are the continuous density and volume. The continuous domain dimensions are computed as presented in section 5.1.1. 


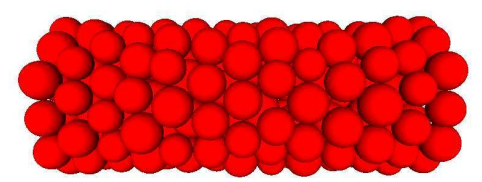

(a) 200

discrete element

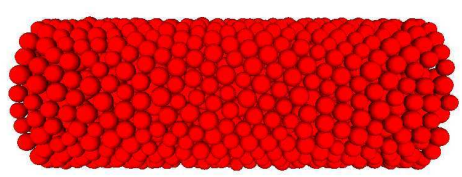

(b) 2000

discrete element

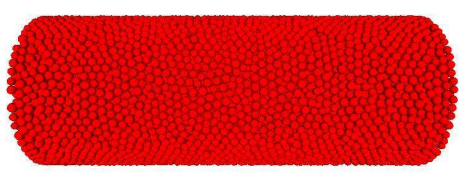

(c) 20000

discrete element

Figure 18: Snapshot of discrete samples with increasing fineness

\begin{tabular}{|c||c|c|c|}
\hline & Tensile & Bending & Torsion \\
\hline \hline Criteria & Free face normal displacement & Free face tangential deflection & Free face rotation \\
\hline Difference & $1.20 \%$ & $4.16 \%$ & $6.13 \%$ \\
\hline
\end{tabular}

Table 3: Comparison of the numerical and theoretical results for the quasi-static tensile, bending and torsion tests

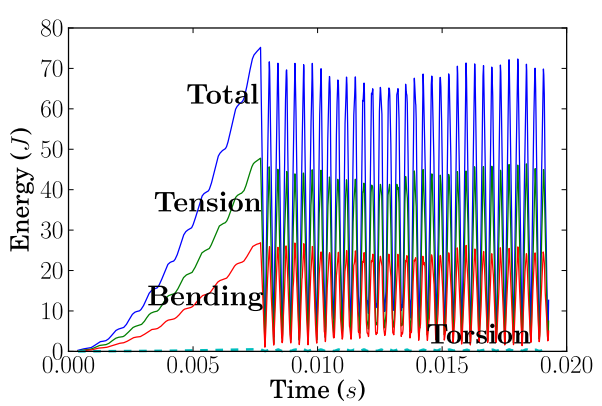

Figure 19: Energy breakdown of total elastic energy stored by cohesive beams for a complex dynamic test (computed with a time step $\Delta t=3 \cdot 10^{-7} \mathrm{~s}$ and a number of iteration it $=50000$ )

However, this method does not ensure inertia equality. This difference is supposed as negligible. This assumption is based on:

1. a classical strength material hypothesis, i.e., for beam transverse vibrations, the cross section rotation energies are negligible compared to the translation energies.

2. the sum of the local torsion energies is negligible in the quasi-static (see figure 12) and dynamic (see figure 19) DEM tests.

This section deals with the validation of this hypothesis by verifying the calibration method by dynamic tensile, bending, torsion and impact tests on the discrete domain (cf figure 9). The numerical results are compared to the analytical results given for the associated continuous domain.

The macroscopic characteristics correspond to silica glass, with a Young's modulus, Poisson's ratio, and density equal to $E_{M}=72.5 \mathrm{GPa}, v_{M}=0.17$ and $\rho_{M}=2201 \mathrm{~kg} / \mathrm{m}^{3}$. The microscopic elastic parameters correspond to those deduced in section 5. The microscopic density is deduced from the calibration method based on mass equality between the discrete and the continuous domains. The continuous domain dimensions are computed by the method presented in section 5.1.1.

For the dynamic tensile, bending and torsion tests, the boundary conditions and loadings are: "xMin" fixed and "xMax" loaded. The force acting on "xMax" is applied gradually and then suddenly set to 0 . This loading allows an excitation of the transient response of the discrete domain.

The oscillation periods are computed from a frequencial analysis (FFT) of the average free face position and angular orientation. The numerical results are compared to the theoretical results given by the vibration of the continuous system analytical models $(40, \S 4)$.

For the impact tests an initial velocity on $\vec{X}$ is applied on the "xMax" face to generate a mechanical shock wave. The "xMin" average velocity on $\vec{X}$ is measured at each time step. So, it is possible to observe the moment when the mechanical wave reaches the "xMin" face (see figure 20). The mechanical wave celerity is deduced from the elapsed time corresponding to the mechanical 


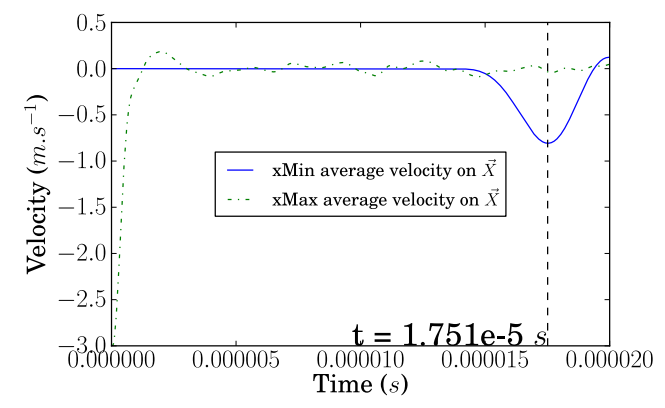

Figure 20: "xMin" and "xMax" face average velocities as a function of time

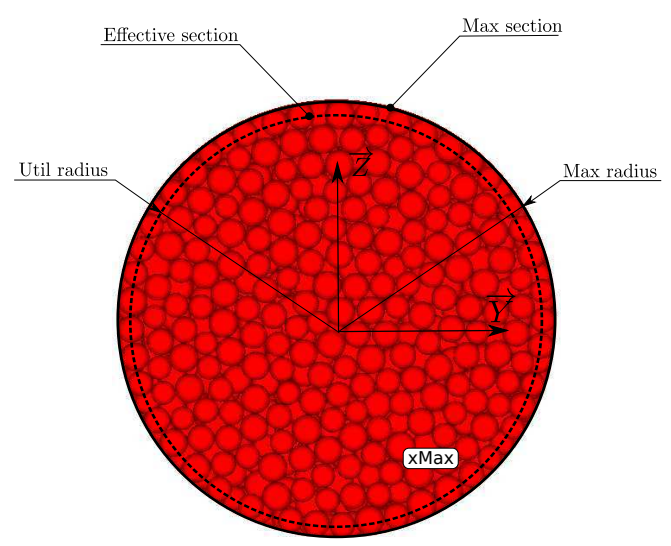

Figure 22: Cross section computational improvement

wave flight between the "xMin" and "xMax" faces (figures 21).

Table 4 shows the comparisons between the simulations and theoretical results. Tensile, torsion and impact tests show a very good agreement with the analytical results. The bending test shows less precision. In fact, the error depends on the cross-sectional moment of inertia computation. In addition, an error in the measurement of the radius has a high influence on results. Figure 22 shows the "xMax" discrete sample face. Following the definition established in section 5.1.1, the sample section is the "Max section". However, the right section, in which internal forces exist, is closer to the "Effective Section". With this new definition of the sample radius, the error between the numerical and the theoretical results for dynamic bending tests is around $1.8 \%$. At the moment, this new definition is too experimental. Its impact on the elastic calibration and the dynamic method needs to be further explored.

\section{Conclusion}

A methodology to verify the initial compact domain using geometrical criteria : the isotropy, the cardinal number, the volume fraction and the fineness has been presented. The most important criterion that further impacts mechanical properties is the geometrical isotropy. An original method, based on geometrical and statistical analysis, is presented to ensure a good level of isotropy. A simple fineness criterion is also presented to ensure a stable geometrical criterion.

A methodology has been presented to obtain the desired value of the Young's modulus and the Poisson's ratio at the macroscopic scale for a 3D spherical discrete element bonded by microscopic beams. This calibration is based on a parametric study of the microscopic and macroscopic elastic parameters. The deduced curves can be used as an "abacus" to calibrate the elastic parameters easily.

A calibration method for the dynamic parameters has been presented. The discrete element density is computed to ensure equality between the continuous and the total discrete domain mass.

The calibration results do not depend on the discrete element size. This important property validates the interest of the micro beam model and the proposed calibration method.

Numerical samples of silica glass has been calibrated. This sample has been tested under tensile, bending, and torsion quasi-static and dynamic tests. The results show good agreement with the strength of material theory.

In conclusion, with the proposed methodology, a discrete element model for homogeneous and isotropic materials is obtained with good quantitative results. DEM has often been used as a qualitative tool to understand complex phenomena such as wear, fracturing or impact. This work is a first step to propose a quantitative numerical tool that will be able to propose predictive models for these classes of problems that have no predictive numerical model presently.

\section{Acknowledgements}

This work is supported by the Conseil Régional d'Aquitaine and is performed in the framework of the Etude et Formation en Surfacage Optique (EFESO) project.

The developments carried out during this project have been implemented in the Granoo Project, see http://www.granoo.org for details. 


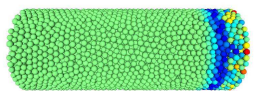

(a) $t \approx 3.8 \mu \mathrm{s}$

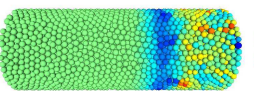

(b) $t \approx 7.6 \mu \mathrm{s}$

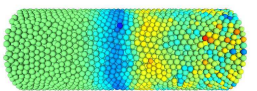

(c) $t \approx 11.4 \mu \mathrm{s}$

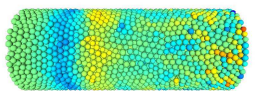

(d) $t \approx 15.2 \mu \mathrm{s}$

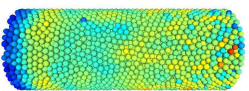

(e) $t \approx 19 \mu \mathrm{s}$

Figure 21: Snapshots of mechanical wave propagation

\begin{tabular}{|c||c|c|c|c|}
\hline & Tensile & Bending & Torsion & Impact \\
\hline \hline Criteria & $\begin{array}{c}\text { Free face normal } \\
\text { oscillation }\end{array}$ & $\begin{array}{c}\text { Free face tangential } \\
\text { oscillation }\end{array}$ & $\begin{array}{c}\text { Free face rotational } \\
\text { oscillation }\end{array}$ & $\begin{array}{c}\text { Mechanical wave } \\
\text { celerity }\end{array}$ \\
\hline Difference & $0.38 \%$ & $6.63 \%$ & $0.50 \%$ & $0.40 \%$ \\
\hline
\end{tabular}

Table 4: Comparison of the numerical and theoretical results for the dynamic tensile, bending, torsion and impact tests

\section{References}

[1] P. A. Cundall, O. D. L. Strack, A discrete numerical model for granular assemblies, Geotechnique 29 (1979) 47-65. doi:10.1680/geot.1979.29.1.47.

[2] S. Hentz, F. V. Donzé, L. Daudeville, Discrete element modelling of concrete submitted to dynamic loading at high strain rates, Computers \& Structures 82 (29-30) (2004) 2509-2524. doi:10.1016/j.compstruc.2004.05.016.

[3] A. Bobet, A. Fakhimi, S. Johnson, J. Morris, F. Tonon, M. R. Yeung, Numerical models in discontinuous media: Review of advances for rock mechanics applications, Journal of Geotechnical and Geoenvironmental Engineering 135 (11) (2009) 1547 1561. doi:10.1061/(ASCE)GT.1943-5606.0000133.

[4] Y. Tan, D. Yang, Y. Sheng, Discrete element method (dem) modeling of fracture and damage in the machining process of polycrystalline sic, Journal of the European Ceramic Society 29 (6) (2009) 1029-1037. doi:DOI 10.1016/j.jeurceramsoc.2008.07.060.

[5] I. Iordanoff, A. Battentier, J. Neauport, J. Charles, A discrete element model to investigate sub-surface damage due to surface polishing, Tribology International 41 (11) (2008) 957-964 doi:10.1016/j.triboint.2008.02.018.

[6] D. Potyondy, P. A. Cundall, A bonded-particle model for rock, International Journal of Rock Mechanics and Mining Sciences 41 (8) (2004) 1329-1364, rock Mechanics Results from the Underground Research Laboratory, Canada. doi:DOI: 10.1016/j.ijrmms.2004.09.011.

[7] S. Hentz, L. Daudeville, F. V. Donzé, Identification and validation of a discrete element model for concret, Journal of Engineering Mechanics 130 (6) (2004) 709-719. doi:10.1061/(ASCE)0733-9399(2004)130:6(709).

[8] A. Fakhimi, T. Villegas, Application of dimensional analysis in calibration of a discrete element model for rock deformation and fracture, Rock Mechanics and Rock Engineering 40 (2) (2007) 193-211. doi:10.1007/s00603-006-0095-6.

[9] F. A. Tavarez, M. E. Plesha, Discrete element method for modelling solid and particulate materials, International Journal for Numerical Methods in Engineering 70 (2007) 379-404. doi: $10.1002 / \mathrm{nme} .1881$.

[10] E. Schlangen, E. Garboczi, Fracture simulations of concrete us- ing lattice models: Computational aspects, Engineering Fracture Mechanics 57 (2-3) (1997) 319-332. doi:10.1016/S00137944(97)00010-6.

[11] E. Schlangen, E. J. Garboczi, New method for simulating fracture using an elastically uniform random geometry lattice, International Journal of Engineering Science 34 (10) (1996) 11311144. doi:DOI: 10.1016/0020-7225(96)00019-5.

[12] H. A. Carmona, F. K. Wittel, F. Kun, H. J. Herrmann, Fragmentation processes in impact of spheres, Physical Review 77 (5) (2008) 051302. doi:10.1103/PhysRevE.77.051302.

[13] A. Munjiza, The combined Finite-Discrete Element Methode, John Wiley \& Sons, 2004. doi:10.1002/0470020180.

[14] O. C. Zienkiewicz, R. L. Taylor, The finite element method for solid and structural mechanics, 6th edition, butterworthheinemann Edition, Elsevier, 2005.

[15] M. Ostoja-Starzewski, Lattice models in micromechanics, Applied Mechanics Reviews 55 (2002) 35-60. doi:10.1115/1.1432990.

[16] H. J. Herrmann, Proceedings of the nato advanced study institute, cargèse, corsica, france, 18-31 july, 1988, in: Random Fluctuations and Pattern Growth: Experiments and Models Random Fluctuations and Pattern Growth: Experiments and Models, Vol. 157 of NATO Science Series E, Stanley, Harry Eugene; Ostrowsky, N., 1988, pp. 149-160.

[17] E. Schlangen, J. G. M. van Mier, Experimental and numerical analysis of micromechanisms of fracture of cement-based composites, Cement and Concrete Composites 14 (2) (1992) 105118, special Issue on Micromechanics of Failure in Cementitious Composites. doi:DOI: 10.1016/0958-9465(92)90004-F.

[18] E. Schlangen, J. G. M. van Mier, Simple lattice model for numerical simulation of fracture of concrete materials and structures, Materials and Structures 25 (9) (1992) 534-542. doi:10.1007/BF02472449.

[19] F. Kun, H. J. Herrmann, A study of fragmentation processes using a discrete element method, Computer Methods in Applied Mechanics and Engineering 138 (1-4) (1996) 3-18. doi:DOI: 10.1016/S0045-7825(96)01012-2.

[20] G. A. D'Addetta, F. Kun, E. Ramm, On the application of a discrete model to the fracture process of cohesive granular materials, Granular Matter 4 (2) (2002) 77-90. doi:10.1007/s10035- 
002-0103-9.

[21] A. Ibrahimbegovic, A. Delaplace, Microscale and mesoscale discrete models for dynamic fracture of structures built of brittle material, Computers \& Structures 81 (21) (2003) 1255-1265. doi:10.1016/S0045-7949(03)00040-3.

[22] G. A. D'Addetta, E. Ramm, A microstructure-based simulation environment on the basis of an interface enhanced particle model, Granular Matter 8 (3) (2006) 159-174. doi:10.1007/s10035-006-0004-4.

[23] S. P. Timoshenko, History of strength of materials: with a brief account of the history of theory of elasticity and theory of structures, Dover, New York, NY.

[24] O. G. Gupta, Finite and boundary element methods in engineering, Belkema, A. A., 1999.

[25] E. Rougier, A. Munjiza, N. W. M. John, Numerical comparison of some explicit time integration schemes used in dem, fem/dem and molecular dynamics, International Journal for Numerical Methods in Engineering 61 (6) (2004) 856-879. doi:10.1002/nme.1092.

[26] D. Eberly, Game physics, second edition Edition, Elsevier, Morgan Kaufmann, 30 Corporate Drive, Suite 400, Burlington, MA 01803 USA, 2010.

[27] T. Pöschel, T. Schwager, Computational granular dynamics, Springer, 2005.

[28] B. D. Lubachevsky, F. H. Stillinger, Geometric properties of random disk packings, Journal of Statistical Physics 60 (1990) 561583. doi:10.1007/BF01025983.

[29] C. L. Martin, D. Bouvard, S. Shima, Study of particle rearrangement during powder compaction by the discrete element method, Journal of the Mechanics and Physics of Solids 51 (4) (2003) 667-693. doi:10.1016/S0022-5096(02)00101-1.

[30] J.-F. Jerier, V. Richefeu, D. Imbault, F.-V. Donzé, Packing spherical discrete elements for large scale simulations, Computer Methods in Applied Mechanics and Engineering 199 (25-28) (2010) 1668-1676. doi:10.1016/j.cma.2010.01.016.

[31] K. Lochmann, L. Oger, D. Stoyan, Statistical analysis of random sphere packings with variable radius distribution, Solid State Sciences 8 (12) (2006) 1397-1413. doi:DOI: 10.1016/j.solidstatesciences.2006.07.011.

[32] J. L. Finney, Random packings and the structure of simple liquids. i. the geometry of random close packing, Proceedings of the Royal Society of London. Series A, Mathematical and Physical Sciences 319 (1539) (1970) pp. 479-493.

[33] K. Gotoh, J. L. Finney, Statistical geometrical approach to random packing density of equal spheres, Nature 252 (1974) 202205. doi:10.1038/252202a0.

[34] B. Cambou, Behaviour of Granular Materials, illustrated edition Edition, Springer, 1998.

[35] B. Cambou, M. Jean, F. Radjai, Micromechanics of Granular Materials, Wiley, 2009.

[36] K. Ken-Ichi, Distribution of directional data and fabric tensors, International Journal of Engineering Science 22 (2) (1984) 149_ 164. doi:10.1016/0020-7225(84)90090-9.

[37] T. Pöschel, S. Luding, Granular Gases, Vol. Vol. 564 of Lecture Notes in Physics, Pöschel, Thorsten; Luding, Stefan, 2001.

[38] L. Mahéo, V. Grolleau, G. Rio, Damping efficiency of the tchamwa-wielgosz explicit dissipative scheme under instantaneous loading conditions, Comptes Rendus Mécanique 337 (1112) (2009) 722-732. doi:doi:10.1016/j.crme.2009.10.005.

[39] C.-L. Liao, T.-P. Chang, D.-H. Young, C. S. Chang, Stress-strain relationship for granular materials based on the hypothesis of best fit, International Journal of Solids and Structures 34 (3132) (1997) 4087-4100. doi:10.1016/S0020-7683(97)00015-2.

[40] A. A. Shabana, Vibration of discrete and continuous systems, 2nd Edition, Mechanical engineerings series, Springer, 1997. 